\title{
REDES SOCIALES, VEJEZ Y POBREZA EN EL DEPARTAMENTO DEL QUINDÍO: El funcionamiento de las redes sociales de personas mayores de 60 años en condición de pobreza
}

\section{SOCIAL NETWORKS, OLD AGE AND POVERTY IN THE DEPARTMENT OF QUINDIO: The functioning of the social networks of the elderly people in poverty.}

\author{
César Augusto Gómez Santos ${ }^{1}$ - Leonardo Iván Quintana Urrea ${ }^{2}$
}

\author{
Fecha de recibido: Septiembre 22 de 2010 \\ Fecha de aceptado: Abril 4 de 2011 \\ Correspondencia: Programa de Gerontología. Universidad del Quindío Av. Bolivar calle 12 norte Armenia Quindío \\ ${ }^{1}$ Programa de Gerontología de la Facultad de Ciencias Humanas y Bellas Artes de la Universidad del Quindío. Miembro del Grupo de Investigación en \\ Desarrollo de la Facultad. Correo electrónico: cegomez@uniquindio.edu.co \\ 2 Programa de Gerontología de la Facultad de Ciencias Humanas y Bellas Artes de la Universidad del Quindío. Miembro del Grupo de Investigación en \\ Desarrollo de la Facultad. Correo electrónico: leonardoivan@yahoo.com
}

\section{RESUMEN}

En este trabajo se da cuenta del funcionamiento de las redes sociales de las personas mayores de 60 años que residen en contextos urbanos y que se encuentran en situación de pobreza o de pobreza extrema. En él se presenta parte de los resultados del proyecto del mismo nombre que ha sido financiado por la Vicerrectoría de investigaciones de la Universidad del Quindío.

La muestra para el estudio ha sido constituida por 591 personas mayores de 60 años que están clasificados en los niveles 1 y 2 del Sistema de Selección de Beneficiarios (SISBEN), a quienes se les ha indagado acerca de la percepción que tienen del funcionamiento de sus redes sociales informales tanto familiares como no familiares, así como los apoyos que reciben y que provienen de las redes formales de apoyo.

Se evidenció a través de la realización de este trabajo que las redes sociales se afectan debido a las precarias condiciones de vida que los adultos mayores presentan, pero que a pesar de ello las transferencias y los apoyos recíprocos ayudan a cubrir algunas de las necesidades que experimenta este grupo poblacional. Se desvirtúa además la falsa creencia de que los adultos mayores juegan un papel pasivo como receptor de ayudas de parte de las redes sociales, contrario a lo que se piensa se constata la reciprocidad de los intercambios de las personas mayores a pesar de las condiciones de pobreza, llegando a ser en muchos casos un proveedor de ayudas hacia los demás miembros de la red social.

Palabras clave: Vejez, envejecimiento, redes sociales, pobreza, apoyo social, apoyo informal, apoyo formal.

\begin{abstract}
This article is about the functioning of social activities that are offered to people over 60 years old residing in urban settings and living in poverty or in extreme poverty. This project presents some of the results of another project named in the same way and financed by the research department of the University of Quindio.
\end{abstract}

The study sample was composed of 591 people over the age of 60 who were classified in levels 1 and 2 of the Selection System of Beneficiaries (SISBEN). They have been inquired about the perception they have of the functioning of the informal support offered not only by their relatives, but also by not family members. Besides, they were asked about the support they receive that comes from formal groups such as institutions and professionals in the field.

It was evidenced through the development of this study that these social groups are affected due to older adults' poor living conditions. However, the transfers and the reciprocal support help fulfill some of the needs this population group may have. It also undermines the false belief that elderly people play a passive role as a recipient of aid from formal social groups. Contrary to what was believed at the beginning of this study, it was evidenced the 
mutual help among the elderly despite the poor conditions, becoming in many cases a supplier of aid to other members of the social group.

Key Words: Oldness, aging, social groups, poverty, social support, informal social support, formal social support.

\section{INTRODUCCIÓN}

La investigación realizada en el Departamento del Quindío ha permitido evidenciar una alta relación entre vejez y pobreza. Según Gómez (2008), la incidencia de pobreza en la población mayor de 60 años medidos por diversos métodos ha dado como resultado niveles exacerbados de pobreza en este grupo poblacional, es así como por Línea de Pobreza (LP) se encontró que el $30,80 \%$ de las personas mayores es pobre y el 56,34 es indigente; por el método de Necesidades Básicas Insatisfechas (NBI) el 27,54\% de la población se clasifica como pobre y el $9,18 \%$ como pobre extremo y finalmente por el Método Combinado de Medición de la Pobreza el 33,33\% se clasifica como en condición de pobreza crónica (pobres por LP y con NBI), el 53,80\% como pobres coyunturales (pobres por LP sin NBI), el $4,68 \%$ en situación de pobreza inercial (no pobres por LP y con NBI) y solamente el $8,19 \%$ presenta una condición de integración social o no pobreza (no pobres por LP y sin NBI). Por otra parte Giraldo et al 2008 demostraron que sin importar la clasificación por NBI, más del $80 \%$ de la población mayor de 60 años en Armenia, presenta dependencia desde el punto de vista de los recursos propios (generados por trabajo, jubilaciones, pensiones u otros).

Los autores de este artículo no pretenden argumentar que exista una relación directa entre vejez y pobreza, afirmación que es frecuente encontrar entre los autores que defienden los enfoques funcionalistas ${ }^{3}$; que tienden a homogeneizar a las personas Adultas Mayores, (en adelante AM) incluso se ha generalizado un estereotipo social que suele identificar y correlacionar ambos términos; en su lugar piensan que en la sociedad quindiana se presenta esta situación entre otras razones porque, ante la inexistencia de las políticas de seguridad social, las personas que a causa de su edad pierden la capacidad de trabajar son víctimas fáciles de la pobreza, es decir, la pobreza es una consecuencia de las condiciones socioeconómicas, políticas y culturales específicas de las personas mayores y no una consecuencia atribuible al simple paso del tiempo. Debe tenerse en cuenta que en nuestro contexto las principales fuentes de soporte económico y protección de los adultos mayores (AM) provienen en primer lugar, de su trabajo $y$, en un segundo lugar, del cuidado familiar o de los amigos, de la caridad privada o de la asistencia pública para pobres.

En la actual sociedad colombiana la vejez es una problemática estrictamente individual o familiar, es decir, forma parte del ámbito privado, tal como lo plantea Minois, 1987; Laslett, 1995 y Johnson, 2002, entre otros. Influye en ello que una elevada proporción de la fuerza de trabajo se mantiene todavía en el sector rural (caracterizado por largas jornadas y mala remuneración), el empleo por cuenta propia -el cual comparte las mismas características descritas para el trabajo rural y además dificulta el acceso a la seguridad social-, es muy significativo en cuanto a la gran proporción de personas mayores que lo realizan y finalmente, que el trabajo asalariado protegido constituye sólo un grupo marginal en el mercado laboral.

Para los autores de este trabajo, un escenario social como el descrito arriba, las redes sociales juegan un papel fundamental para el bienestar de las personas mayores, dado que es a través de ellas que se activa un flujo de apoyos sociales de carácter formal e informal, ellas se convierten en una fuente de apoyo para las personas mayores; queda manifiesto entonces que las redes son expresiones de construcción del capital social ${ }^{4}$ de los grupos poblacionales y el de las personas mayores no es la excepción.

\footnotetext{
${ }^{3}$ Como por ejemplo Rose $(1965 ; 1962)$, G, Streib, citado por Bazo, (1990)

${ }^{4}$ Lindon; Siles y Schmid, (2003) definen el capital social como los sentimientos de solidaridad de una persona o un grupo por otra persona o grupo. Esos sentimientos pueden abarcar la admiración, el interés, la preocupación, la empatía, la consideración, el respeto, el sentido de obligación o la confianza respecto de otra persona o grupo. Este concepto de capital social sugiere que existen diversas clases de intensidades de relaciones de solidaridad. Por ejemplo, hay un tipo de capital social que se basa en el afecto y la preocupación por el otro. Existe otra clase de capital social que se basa en los sentimientos de compañerismo y buena voluntad recíproca que pueden existir entre personas de la misma condición e iguales recursos. Finalmente, hay un tipo de capital social que se basa en los sentimientos de respeto o conciencia de la existencia del otro que puede haber entre personas que mantienen una relación asimétrica de poder e influencia. Para poder distinguir entre las diversas clases de capital social, los autores las definen como capital social de unión o lazos (bonding social capital), de vinculación o escalera (linking social capital) y de aproximación o puente (bridging social capital).
} 
El apoyo social se entiende "como las transacciones interpersonales que implican ayuda, afecto y afirmación" Khan y Antonucci (1980). También son denominados como transferencias, que se presenta como un flujo de recursos, acciones e información que se intercambia y circula.

Desde la perspectiva de Guzmán, Huenchuan y Montes de Oca (2003) la categoría "redes sociales" ha sido asumida como indicador de apoyo, debido a que se asume que si se pertenece a una red se está apoyado. Aunque en las investigaciones gerontológicas se evidencia que esto no es del todo cierto, por esta razón se inició por parte de algunos teóricos un análisis más detallado de la calidad, frecuencia, efectividad y disponibilidad de los apoyos, el presente estudio se emprendió precisamente para procurar aportar respuestas a este propósito.

En el abordaje del estudio de las redes sociales se reconocen dos corrientes teóricas que han desarrollado el concepto: la anglosajona ${ }^{5}$ y la latinoamericana. Para efectos de este trabajo los autores se han orientado por la segunda corriente teórica, la cual ha aportado a la conceptualización de la temática de las redes sociales y que inició desde la perspectiva antropológica. Para los autores que se inscriben en esta vertiente el énfasis del análisis estuvo centrado en la importancia de las redes sociales en las estrategias de reproducción social de aquellos que se encontraban en situación desventajosa (migrantes, sectores populares, jefatura femenina, desempleados, madres solteras, entre otros).

Dentro de los países que más han trabajado la temática están México y Argentina. Una de las más destacadas autoras mexicanas es Lomnitz (1975, p. 26) quien hizo una aproximación al tema y determinó que "las redes de intercambio desarrolladas por los pobladores, constituyen un mecanismo efectivo para suplir la falta de seguridad social, reemplazandola con un tipo de ayuda mutua basado en la reciprocidad". A los que luego agregó que también permiten ubicar los vínculos verticales y las relaciones de apoyo existentes entre grupos empresariales, comunidades científicas y en lo que ella denominó, redes informales en sistemas formales.

Por su parte, González de la Rocha (1999) retomando los planteamientos de Mingione (1994), argumenta que las redes sociales actúan en contextos y grupos sociales específicos y presentan diferencias de acuerdo al género. Además se basan en principios de reciprocidad diferencial que permite la continuidad y la permanencia de las relaciones sociales.

Sumado a esto De la Peña (2001) plantea que en cierta medida el que exista una red es muestra de que no hay igualdad de oportunidades para todos los grupos sociales y es en la búsqueda de beneficios de los más desposeídos, donde las redes se estructuran y reestructuran para conservar o aumentar los recursos.

De otro lado, en el caso argentino Dabas (1993) sustenta que los movimientos sociales personificados en redes sociales definidas por la autora como redes comunitarias dan solución a demandas sociales específicas.

Estas redes comunitarias tienen diferentes implicaciones que se perciben de manera colectiva. Se reconoce la construcción de vínculos subjetivos que están determinados según la autora por el hecho de "haber participado", "haber logrado", "haber compartido" y que propenden por un empoderamiento por parte de los miembros de la red.

Las situaciones adversas que afrontan varios de los sectores sociales de América Latina según Lomnitz, (1994) demuestran que las redes - sobre todo las redes de reciprocidad - desempeñan un papel importante en los sectores desfavorecidos, al crear un "sistema informal de seguridad social para la supervivencia" que tiende a satisfacer aquellas necesidades no cubiertas por el sistema formal. Para la autora, el intercambio recíproco surge en respuesta a la escasez y se constituye en un sistema de solidaridad mutua esencial, el cual se expresa a través de la reciprocidad, definida como una forma de intercambio de bienes y servicios que tiene las siguientes características: Se desarrolla como parte de una relación social; constituye un flujo recíproco de bienes materiales y servicios que persiste más allá de una sola transacción y no está regido por las leyes de la oferta y la demanda. Las redes actúan de acuerdo con Lomnitz como un seguro colectivo contra las amenazas del sistema formal y como una reserva de recursos, particularmente durante las emergencias.

\footnotetext{
${ }^{5}$ Esta corriente está representada por una importante diversidad autores, algunos de ellos con estudios considerados clásicos, como los de Elizabeth Bott (1957, 1980, 1990); Barnes, J. A.1954; Chappel, N. (1992); Scott,y Wenger , (1996); Lopata (1979); Cobb 1976; citado en Chappell, (1992); Gottlieb (1983).
}

Rev. Invest. Univ. Quindío (22): 30-47. Armenia - Colombia 
Es importante resaltar que no solo la necesidad material es el motor en la constitución de una red, también lo son las necesidades emocionales y cognitivas sobre todo para los adultos mayores. Guzmán, Huenchuan y Montes de oca (2003).

En relación con el desarrollo teórico del tema Colombia, se puede afirmar que son escasos los trabajos relacionados con el tema de las redes sociales, se puede citar el trabajo titulado “Relación entre la Pertenencia a Redes Sociales y la Dinámica Familiar de mujeres en condiciones de Pobreza“, realizado por Omar Sierra García, Camilo Madariaga Orozco y Raimundo Abello Llanos, en el cual se describe la red como un mecanismo de supervivencia de familias pobres, pero que incide también positivamente en la dinámica de las mismas.

A pesar del desarrollo teórico en el tema de las redes sociales, actualmente no se cuenta con un concepto exacto y unificado de estas, por esta razón los autores de este artículo se inclinan por los planteamientos de Guzmán, Huenchuan y Montes de Oca (2003, p. 3) quienes definen las redes sociales como "una práctica simbólica-cultural que incluye el conjunto de relaciones interpersonales que integran a una persona con su entorno social y le permite mantener o mejorar su bienestar material, físico y emocional y evitar así el deterioro real o imaginado que podría generarse cuando se producen dificultades, crisis o conflictos que afectan al sujeto".

Tal como se planteó anteriormente, este trabajo pretende aportar al estudio y conceptualización de las redes sociales en la población adulta mayor, a través de la socialización de una parte de los resultados obtenidos de la ejecución del proyecto de investigación número 370 titulado "REDES SOCIALES DE LA POBLACIÓN MAYOR DE 60 AÑOS EN CONTEXTOS URBANOS DE POBREZA DEL DEPARTAMENTO DEL QUINDIO". Este artículo brinda la información necesaria para responder puntualmente al objetivo

- Analizar el funcionamiento de las redes sociales a partir de las funciones de apoyo, orientación, compañía social, control, conectividad o guía y promotor de nuevos contactos.

Este proyecto ha sido financiado por la Vicerrectoría de Investigaciones de la Universidad del Quindío y se ejecutó entre agosto de 2006 y diciembre de 2007.

\section{METODOLOGÍA}

Analizar la estructura y funcionamiento de las redes sociales de las personas mayores de 60 años en relación con las condiciones de vida en contextos urbanos de pobreza del Departamento del Quindío, fue el objetivo central de la investigación, para ello resultó pertinente conocer y analizar las condiciones de vida de las personas mayores de 60 años en relación con las redes sociales y analizar las diferencias y semejanzas de las redes sociales en contextos de pobreza entre hombres y mujeres adultas mayores.

La población objeto de estudio para este trabajo la conformó los adultos mayores de 60 años de la zona urbana del Departamento del Quindío que residan en contextos de pobreza. Para este fin se constituyo como población a los adultos mayores de 60 años que han sido identificados como pobres, (clasificados en el nivel 1 ó 2), mediante el Sistema de Selección de Beneficiarios (SISBEN)

EI SISBEN es un índice que valora una serie de variables cualitativas que reflejan o expresan las condiciones de vida de los hogares urbanos y rurales Colombianos

Los puntajes del índice se agrupan en seis niveles, definidos de acuerdo con la probabilidad de que expresen situaciones de pobreza o rangos de ingreso por persona de las familias.

Los dos primeros niveles se asocian con grados de intensidad de la pobreza, de acuerdo con el uso simultáneo de los métodos de ingresos - LP- y NBI. EI nivel 1 está correlacionado con circunstancias de las familias en pobreza extrema: con dos o más indicadores de NBI (miseria) o con ingresos inferiores a la línea de indigencia (valor de una canasta básica de alimentos). El nivel 2 tiende a captar las restantes características de la pobreza identificadas por los dos métodos.

La selección de los sujetos se realizó mediante un muestreo probabilístico estratificado aleatorio, el tamaño de la muestra fue de 591 personas mayores de 60 años, de los cuales 233 eran hombres 358 eran mujeres cuyas edades oscilaron entre los 60 y los 84 años. El error muestral fue de 0.04 , esto quiere decir que existe un error potencial del $4 \%$, la confiabilidad de la muestra es del $95 \%$.

Como técnica de recolección de la información se utilizó el cuestionario, para lo cual se diseñó un instrumento 
en el que se indagó sobre los indicadores centrales de la estructura y funcionamiento de las redes sociales y de apoyo. El cuestionario se orientó a explorar las redes sociales de los adultos mayores que conformaron la muestra, éste se dividió en 3 secciones que iban orientadas a indagar sobre la red informal (familiar y no familiar) y la red formal (institucional). Aquí se entiende como red social, a un sistema de apoyo primario integrado al dar y recibir objetos, servicios, apoyo social, emocional, informacional y sentimental. La red informal la constituyen los contactos y consecuentes apoyos provenientes de familiares, vecinos y amigos, mientras que la red formal hace referencia a los contactos y apoyos de instituciones, organizaciones y profesionales.

Las diferentes secciones estaban orientadas a indagar aspectos inherentes a la estructura de las redes sociales como son: Tamaño de la red, densidad, conectividad, composición o distribución de los vínculos, dispersión de la red (ubicación geográfica) y homogeneidad o heterogeneidad entre los distintos sujetos que conforman la red; desde el punto de vista de las funciones de la red se indagó por aspectos tales como la compañía social, el apoyo emocional, el desempeño como guía cognitiva y orientadora, el papel en la regulación y control social, el apoyo en lo material, en lo informacional y en servicios y por último el acceso a nuevos contactos. En cuanto a atributos del vínculo se procuró establecer las funciones centrales del mismo, la frecuencia de los contactos y finalmente los tipos de contacto que se presentan en dichas redes.

\section{RESULTADOS}

\section{El funcionamiento de la red familiar}

En lo concerniente al funcionamiento de las redes sociales de las personas mayores de 60 años se ha encontrado que:

El apoyo social dentro del nucleo familiar se caracteriza porque los adultos mayores son receptores de ayuda en una gran proporción, el $\mathbf{9 7 . 4 8 \%}$ de los integrantes de la red familiar expresaron brindar algún tipo de apoyo al adulto mayor, no se aprecian diferencias en las proporciones por sexo, se aprecia, tanto en el caso de los hombres como el de las mujeres, que la familia se consolida como una fuente importante de soporte para la población adulta mayor (Tabla 1). Independientemente del tamaño de la red, los resultados demuestran la presencia de la red familiar para proveer apoyo a los adultos mayores en contextos de pobreza en el área urbana, se confirma la importancia del papel que juega la familia para que el adulto mayor pueda sobrevivir en las condiciones de pobreza que caracterizan a los sujetos estudiados.

Tabla 1. Recepción de apoyo red familiar

* En esta tabla se representa la percepción del adulto mayor hacia cada uno de los miembros de su red familiar, por esta razón el total supera el tamaño de la muestra.

\begin{tabular}{lcccccc}
\hline Recepción & \multicolumn{2}{c}{ HOMBRE } & \multicolumn{2}{c}{ MUJER } & \multicolumn{2}{c}{ TOTAL } \\
\cline { 2 - 7 } de apoyo & Frec. & $\%$ & Frec. & $\%$ & Frec. & $\%$ \\
\hline SI & 746 & 97,39 & 915 & 97,55 & 1661 & 97,48 \\
NO & 18 & 2,35 & 19 & 2,03 & 37 & 2,17 \\
NS / NR & 2 & 0,26 & 4 & 0,43 & 6 & 0,35 \\
TOTAL & $\mathbf{7 6 6}$ & $\mathbf{1 0 0}$ & $\mathbf{9 3 8}$ & $\mathbf{1 0 0}$ & $\mathbf{1 7 0 4 *}$ & $\mathbf{1 0 0}$ \\
\hline
\end{tabular}

Las ayudas que reciben los adultos mayores (AM) son básicamente de tipo material, instrumental, emocional y cognitivo. Al observar de manera independiente cada uno de estos tipos de ayuda se evidencia que desde el punto de vista material los principales aportes de los miembros de la famillia hacia el adulto mayor lo constituyen las ayudas en dinero, alimentos y un procentaje relativamente más bajo recibe ayuda material a través del pago de los servicios públicos por parte de alguno de los miembros de la famillia (18,60\%). Contrario a lo que podría pensarse los apoyos de tipo material no constituyen el principal aporte de la familia hacia este (esto podría estar determinado por la precariedad social y la misma condición de pobreza de la red familiar), pues de acuerdo con los resultados, desde la perspectiva de los adultos mayores, los apoyos de tipo emocional constituyen el principal aporte que la familia le otorga a sus miembros de mayor edad.

La familia también proporciona apoyos de tipo instrumental cuando las circunstancias así lo requieren, es así como el $29,59 \%$ de los adultos mayores 
expresaron recibir cuidados de su familia y un $18,18 \%$ de los mismos expresó que la familia le proporciona ayuda para realizar las actividades del cuidado del hogar.

La recepción de apoyo de tipo informacional al interior de las redes familiares lo constituyen básicamente los consejos, la recepción de información y el intercambio de experiencias.
En relación con el género se puede afirmar en términos generales que las mujeres reciben mayor cantidad de apoyo que los hombres practicamente en todos los tipos de apoyo, con excepción de algunos de los apoyos de tipo material, como en la recepción de dinero y de ayudas en el pago de servicios (Tabla 2)

Tabla 2. Tipo de apoyo familiar que recibe el Adulto Mayor

\begin{tabular}{lcccccc}
\hline \multirow{1}{*}{ Tipo de apoyo } & \multicolumn{2}{c}{ 1. HOMBRE } & \multicolumn{2}{c}{ 2. MUJER } & \multicolumn{2}{c}{ TOTAL } \\
\cline { 2 - 7 } & Frec. & \% & Frec. & \% & Frec. & \% \\
\hline Dinero & 338 & 54,34 & 284 & 45,66 & 622 & 37.51 \\
Alojamiento & 93 & 44,71 & 115 & 55,29 & 208 & 12.52 \\
Comida & 264 & 47,57 & 291 & 52,43 & 555 & 33.41 \\
Ropa & 104 & 46,64 & 119 & 53,36 & 223 & 13.43 \\
Servicios & 179 & 57,93 & 130 & 42,07 & 309 & 18.60 \\
Cuidado & 177 & 36,12 & 313 & 63,88 & 491 & 29.56 \\
Transporte & 27 & 52,94 & 24 & 47,06 & 51 & 3.07 \\
Cuidados del hogar & 61 & 20,33 & 239 & 79,67 & 302 & 18.18 \\
Afecto & 485 & 43,73 & 624 & 56,27 & 1114 & 67.07 \\
Compañía & 437 & 41,78 & 609 & 58,22 & 1050 & 63.21 \\
Reconocimiento & 204 & 44,16 & 258 & 55,84 & 462 & 27.81 \\
Escucha & 269 & 42,77 & 360 & 57,23 & 632 & 38.05 \\
Intercambio experiencias & 158 & 42,02 & 218 & 57,98 & 377 & 22.70 \\
Información & 168 & 41,69 & 235 & 58,31 & 404 & 24.32 \\
Consejos & 186 & 37,80 & 306 & 62,20 & 492 & 29.62 \\
\hline
\end{tabular}

El adulto mayor no solamente percibe apoyo de parte de los miembros de su familia sino que en una importantísima proporción, también éstos proveen apoyo a los demás miembros de su familia, los resultados demuestran que el $91,26 \%$ de los que conformaron la muestra expresaron brindar algún tipo de apoyo y un escaso 7,98\% expresaron no hacerlo. (Tabla 3). Tampoco se evidencian diferencias en relación con el sexo en esta variable, tanto los hombres como las mujeres proveen ayuda a los miembros de su red familiar.

Tabla 3. Apoyo familiar brindado por el Adulto Mayor

*En esta tabla se representa la percepción del adulto mayor hacia cada uno de los miembros de su red familiar, por esta razón el total supera el tamaño de la muestra.

\begin{tabular}{lcccccc}
\hline \multirow{2}{*}{$\begin{array}{c}\text { Apoyo } \\
\text { brindado }\end{array}$} & \multicolumn{2}{c}{ HOMBRE } & \multicolumn{2}{c}{ MUJER } & \multicolumn{2}{c}{ TOTAL } \\
\cline { 2 - 7 } & Frec. & \% & Frec. & \% & Frec. & $\%$ \\
\hline SI & 704 & 91,91 & 851 & 90,72 & 1555 & 91,26 \\
NO & 58 & 7,57 & 78 & 8,32 & 136 & 7,98 \\
NS / NR & 4 & 0,52 & 9 & 0,96 & 13 & 0,76 \\
TOTAL & $\mathbf{7 6 6}$ & $\mathbf{1 0 0}$ & $\mathbf{9 3 8}$ & $\mathbf{1 0 0}$ & $\mathbf{1 7 0 4 *}$ & $\mathbf{1 0 0}$ \\
\hline
\end{tabular}

El tipo de apoyo que más provee el adulto mayor hacia los diferentes miembros de su familia lo constituye el apoyo de tipo emocional, una alta proporción de ellos considera que brinda afecto y compañía, otro apoyo de carácter emocional importante lo constituye la escucha con un 39,55\%. Brindar consejo es un apoyo de carácter informacional que lo ofrece el $48,36 \%$ de los miembros de la muestra, bajo esa misma tipología de apoyo se encuentra el intercambio de experiencias con una proporción de $27,65 \%$ (Tabla 4).

Rev. Invest. Univ. Quindío (22): 30-47. Armenia - Colombia 
Tabla 4. Tipo de apoyo familiar que brinda el A.M. a la familia

\begin{tabular}{lcccccc}
\hline \multirow{2}{*}{$\begin{array}{c}\text { Tipo de apoyo que } \\
\text { brinda }\end{array}$} & \multicolumn{2}{c}{ HOMBRE } & \multicolumn{2}{c}{ MUJER } & \multicolumn{2}{c}{ TOTAL } \\
\cline { 2 - 7 } & Frec. & $\%$ & Frec. & $\%$ & Frec. & $\%$ \\
\hline Dinero & 40 & 28,99 & 98 & 71,01 & 141 & 9.07 \\
Alojamiento & 109 & 41,76 & 152 & 58,24 & 262 & 16.85 \\
Comida & 131 & 45,64 & 156 & 54,36 & 289 & 18.59 \\
Ropa & 17 & 31,48 & 37 & 68,52 & 54 & 3.47 \\
Servicios & 35 & 29,91 & 82 & 70,09 & 118 & 7.59 \\
Cuidado & 191 & 49,48 & 195 & 50,52 & 389 & 25.02 \\
Transporte & 3 & 16,67 & 15 & 83,33 & 20 & 1.29 \\
Hogar & 198 & 50,77 & 192 & 49,23 & 392 & 25.21 \\
Afecto & 515 & 45,49 & 617 & 54,51 & 1136 & 73.05 \\
Compañía & 394 & 44,17 & 498 & 55,83 & 895 & 57.56 \\
Reconocimiento & 186 & 44,93 & 228 & 55,07 & 417 & 26.82 \\
Escucha & 283 & 46,32 & 328 & 53,68 & 615 & 39.55 \\
Intercambio experiencias & 193 & 45,09 & 235 & 54,91 & 430 & 27.65 \\
Información & 150 & 44,38 & 188 & 55,62 & 340 & 21.86 \\
Consejos & 350 & 46,79 & 398 & 53,21 & 752 & 48.36 \\
No sabe / No responde & 4 & 57,14 & 3 & 42,86 & 7 & 0.45 \\
\hline
\end{tabular}

La condición de pobreza que caracteriza el grupo obstaculiza enormemente la posibilidad de proveer apoyos de tipo material, no obstante se evidencia que en proporciones importantes el A.M. brinda a sus familiares apoyo a manera de alojamiento $(16,85 \%)$ y alimentación (18,59\%).
El apoyo instrumental constituye otro de los aportes importantes que otorga el A.M. a su familia, este se ve representado a través del cuidado que le brinda a los miembros más pequeños del hogar y a aquellos que presentan dependencia física y funcional $(25,02 \%)$ y el cuidado del hogar, representado en oficios domésticos en el $(25,21 \%)$ de los casos.

Tabla 5. Momentos en los que el A.M. recibe mayor apoyo

\begin{tabular}{lcccccc}
\hline \multirow{2}{*}{$\begin{array}{c}\text { Momentos en que } \\
\text { recibe apoyo }\end{array}$} & \multicolumn{2}{c}{ HOMBRES } & \multicolumn{2}{c}{ MUJERES } & \multicolumn{2}{c}{ TOTAL } \\
\cline { 2 - 7 } & Frec. & $\%$ & Frec. & $\%$ & Frec: & $\%$ \\
\hline Separación & 1 & 50,00 & 1 & 50,00 & 2 & 0,34 \\
Enfermedad & 62 & 43,66 & 80 & 56,34 & 142 & 24,03 \\
Muerte & 1 & 20,00 & 4 & 80,00 & 5 & 0,85 \\
Calamidad & 11 & 64,71 & 6 & 35,29 & 17 & 2,88 \\
Dificultades & & & & & & \\
Económicas & 11 & 55,00 & 9 & 45,00 & 20 & 3,38 \\
Soledad & 8 & 33,33 & 16 & 66,67 & 24 & 4,06 \\
Siempre & 139 & 36,48 & 242 & 63,52 & 381 & 64,47 \\
Otro & 3 & 75,00 & 1 & 25,00 & 4 & 0,68 \\
No sabe / No & & & & & & \\
responde & 6 & 42,86 & 8 & 57,14 & 14 & 2,37 \\
\hline
\end{tabular}

Los sujetos que conformaron la muestra expresaron recibir apoyo de parte de los miembros de la red familiar así: el 64,47\% de ellos recibe apoyo siempre, no hace falta que suceda nada extraordinario para que reciban ayuda; el $24,03 \%$ dijo recibir mayor ayuda en los momentos de enfermedad; el 4,06\% cuando está en situación de soledad, el 3,38\% en momentos de crisis económicas; el $2,88 \%$ en calamidades, entre otros porcentajes menos importantes porcentualmente. (Tabla 5)

Los adultos mayores expresaron dar apoyo "siempre" en el $68,53 \%$ de los casos; en momentos de enfermedad el $14,55 \%$, en momentos de soledad el $6,09 \%$ y el $3,55 \%$ en momentos de calamidad. 
Tabla 6. Momentos en los que el A.M. brinda mayor apoyo

\begin{tabular}{|c|c|c|c|c|c|c|}
\hline \multirow{2}{*}{$\begin{array}{l}\text { Momentos que } \\
\text { brinda apoyo }\end{array}$} & \multicolumn{2}{|c|}{ HOMBRES } & \multicolumn{2}{|c|}{ MUJERES } & \multicolumn{2}{|c|}{ TOTAL } \\
\hline & Frec. & $\%$ & Frec. & $\%$ & Frec. & $\%$ \\
\hline Separación & 0 & 0,00 & 2 & 100 & 2 & 0,34 \\
\hline Enfermedad & 38 & 44,19 & 48 & 55,81 & 86 & 14,55 \\
\hline Muerte & 2 & 50,00 & 2 & 50,00 & 4 & 0,68 \\
\hline $\begin{array}{l}\text { Calamidad } \\
\text { Dificultades }\end{array}$ & 10 & 47,62 & 11 & 52,38 & 21 & 3,55 \\
\hline Económicas & 7 & 70,00 & 3 & 30,00 & 10 & 1,69 \\
\hline Soledad & 12 & 33,33 & 24 & 66,67 & 36 & 6,09 \\
\hline Siempre & 145 & 35,80 & 260 & 64,20 & 405 & 68,53 \\
\hline Otro & 6 & 54,55 & 5 & 45,45 & 11 & 1,86 \\
\hline NS / NR & 11 & 50,00 & 11 & 50,00 & 22 & 3,72 \\
\hline
\end{tabular}

Tanto en el acto de dar apoyo como de recibir, se percibe un mayor flujo de ayudas en los momentos en los que el A.M. (en el caso de recibir ayuda) o uno de sus familiares (en el caso de proveer ayuda), se encuentra en situación de enfermedad, en las otras condiciones se presta y se recibe ayuda, aunque no en proporciones tan importantes como la citada.

Tabla 7. Satisfacción con la comunicación con los miembros de la red familiar

\begin{tabular}{lcccccc}
\hline \multirow{2}{*}{$\begin{array}{c}\text { Satisfacción con la } \\
\text { comuni cación }\end{array}$} & \multicolumn{2}{c}{ 1. HOMBRE } & \multicolumn{2}{c}{ 2. MUJER } & \multicolumn{2}{c}{ TOTAL } \\
\cline { 2 - 7 } & Frec. & \% & Frec. & \% & Frec. & \% \\
\hline Muy alta & 196 & 25,5 & 252 & 26,87 & 448 & 26,29 \\
Alta & 297 & 38,7 & 362 & 38,59 & 659 & 38,67 \\
Ni alta ni baja & 208 & 27,1 & 245 & 26,12 & 453 & 26,58 \\
Baja & 35 & 4,57 & 36 & 3,84 & 71 & 4,17 \\
Muy baja & 19 & 2,48 & 14 & 1,49 & 33 & 1,94 \\
NS/ NR & 11 & 1,44 & 29 & 3,09 & 40 & 2,35 \\
TOTAL & $\mathbf{7 6 6}$ & $\mathbf{1 0 0}$ & $\mathbf{9 3 8}$ & $\mathbf{1 0 0}$ & $\mathbf{1 7 0 4}$ & $\mathbf{1 0 0}$ \\
\hline
\end{tabular}

La satisfacción en la comunicación entre los miembros de la red familiar es muy alta en el $26,29 \%$ de los casos y alta en el $38,67 \%$, estos índices permiten concluir que el nivel de comunicación entre los miembros de la familia con el adulto mayor son adecuados dado que el $64,96 \%$ se ubican dentro de los dos rangos de mayor satisfacción y $26,58 \%$ presenta un grado de satisfacción aceptable.

Un indicador del grado de densidad y conectividad entre los integrantes de la red familiar, lo constituye el hecho de que se hayan disuelto o no contactos en el último mes, los resultados indican que esto no ha sucedido en el $81.73 \%$ de los casos, lo que sugiere que las relaciones entre los miembros de la familia con el adulto mayor muestran ser muy estables, esto a su vez puede ser visto como una fortaleza, siendo consecuentes con lo planteado en relación con la importancia que tiene la red familiar para estas personas en contextos de pobreza.

Tabla 8. Disolución de contactos con los miembros de la red familiar

\begin{tabular}{lcccccc}
\hline \multicolumn{1}{c}{$\begin{array}{c}\text { Disolución de } \\
\text { contactos }\end{array}$} & \multicolumn{2}{c}{ HOMBRES } & \multicolumn{2}{c}{ MUJERES } & \multicolumn{2}{c}{ TOTAL } \\
\cline { 2 - 7 } & Frec. & $\%$ & Frec. & \% & Frec. & $\%$ \\
\hline SI & 24 & 10,30 & 35 & 9,78 & 59 & 9,98 \\
NO & 184 & $\mathbf{7 8 , 9 7}$ & 298 & 83,2 & 482 & 81,56 \\
NS/NR & 25 & 10,73 & 25 & 6,98 & 50 & 8,46 \\
TOTAL & $\mathbf{2 3 3}$ & $\mathbf{1 0 0}$ & $\mathbf{3 5 8}$ & $\mathbf{1 0 0}$ & $\mathbf{5 9 1}$ & $\mathbf{1 0 0}$ \\
\hline
\end{tabular}


De nuevo se aprecia que las diferencias en las proporciones entre hombres y mujeres no son porcentualmente significativas.

\section{El funcionamiento de la red no familiar}

La red social informal está constituida por los vínculos personales con vecinos y amigos, con las redes comunitarias no estructuradas como programas de apoyo y en general con contactos que se tienen en la comunidad. En este apartado se provee información que permite apreciar el funcionamiento de la red informal de las personas mayores desde la perspectiva de los mismos sujetos, dicho de otra manera se presenta la percepción de apoyo recibido y brindado que tiene la persona mayor de su red informal, para este trabajo no se indagó el punto de vista de los miembros que conforman la red informal.

Lo primero que se debe advertir es que mas de la mitad de los miembros de la muestra, esto es el 52,62\% o 311 sujetos (Tabla 9), expresaron no tener red informal no familiar (RINF), estos sujetos expresaron no tener ni amigos ni vecinos dentro de su círculo relacional tampoco participan de actividades ni de grupos dentro de su comunidad, lo cual demuestra la preocupante reducción de los vínculos de los adultos mayores en condición de pobreza, situación que lo conduce al aislamiento social y reafirma de alguna manera que la carencia de recursos limita las interacciones actuales así como las potenciales o futuras toda vez que ellas pueden comprometer recursos en su mantenimiento.
Tabla 9. Tamaño de la red no familiar (Vecinos y amigos)

\begin{tabular}{ccc}
\hline $\begin{array}{c}\text { No. De } \\
\text { amigos }\end{array}$ & TOTAL & $\%$ \\
\hline $\mathbf{0}$ & $\mathbf{3 1 1}$ & 52,62 \\
$\mathbf{1}$ & 136 & 23,01 \\
$\mathbf{2}$ & 68 & 11,51 \\
$\mathbf{3}$ & 36 & 6,09 \\
$\mathbf{4}$ & 25 & 4,23 \\
$\mathbf{5}$ & 5 & 0,85 \\
$\mathbf{6}$ & 3 & 0,51 \\
$\mathbf{7}$ & 2 & 0,34 \\
$\mathbf{8}$ & 1 & 0,17 \\
$\mathbf{9}$ & 3 & 0,51 \\
$\mathbf{1 0}$ & 0 & 0,00 \\
$\mathbf{1 1}$ & 0 & 0,00 \\
$\mathbf{1 2}$ & 1 & 0,17 \\
TOTAL & $\mathbf{5 9 1}$ & $\mathbf{1 0 0 , 0 0}$ \\
\hline
\end{tabular}

La información que aparece en las tablas de ésta sección corresponden a datos de los miembros que conforman la red social informal del 47,38\% (280 casos) de los sujetos que expresaron SI poseer RINF, los cuales suman 584 , esto permitirá apreciar la percepción del adulto mayor de las proporciones de recepción y otorgamiento de apoyos sociales.

Las RINF juegan un papel importante en la provisión de apoyo a las generaciones más viejas, los datos permiten evidenciar que una alta proporción de amigos, entre quienes tienen (RINF), le proveen apoyo o ayuda de diversa naturaleza a los adultos mayores (90,41\%), mientras que un escaso $8,73 \%$ expresó no recibir ningún tipo de apoyo de parte de sus amigos, (Tabla 10).

Tabla 10. Recepción de apoyo no familiar (Amigos)

\begin{tabular}{lcccccc}
\hline \multirow{2}{*}{$\begin{array}{c}\text { Recepción } \\
\text { de apoyo }\end{array}$} & \multicolumn{2}{c}{ HOMBRES } & \multicolumn{2}{c}{ MUJERES } & \multicolumn{2}{c}{ TOTAL } \\
\cline { 2 - 7 } Frec. & $\%$ & Frec. & $\%$ & Frec. & $\%$ \\
\hline SI & 161 & 91,48 & 367 & 89,95 & 528 & 90,4 \\
NO & 11 & 6,25 & 40 & 9,80 & 51 & 8,73 \\
NS/ NR & 4 & 2,27 & 1 & 0,25 & 5 & 0,86 \\
\hline
\end{tabular}

Del análisis de la información suministrada por los 280 sujetos que tienen RINF en cuanto al tipo de apoyo, se ha encontrado que la red no familiar no otorga ayudas de tipo material, o por lo menos no de manera tan importante como lo hace la red familiar; mientras que el cuidado que hace parte del apoyo de tipo instrumental se brinda, en el $21,97 \%$ del total de la muestra, porcentaje muy próximo al valor obtenido en la red familiar; por otra parte los apoyos de tipo emocional (afecto $34,66 \%$, compañía $71,59 \%$, escucha $46,40 \%$ y reconocimiento $21,21 \%$ ) y los de tipo cognitivo (intercambio de experiencias $42,23 \%$, de información $35,23 \%$, y los consejos $30,11 \%$ ) constituyen los principales aportes de los amigos hacia los adultos mayores.

Las altas proporciones en que se ofrecen los tipos de apoyo citados atrás, brindan una idea clara de la importancia del tema de la red informal no familiar y del papel que cumplen los amigos y vecinos en la vida social de los adultos mayores del departamento. 
Gómez S., C.A., Quintana U., L.I. - 39 -

Tabla 11. Tipo de apoyo no familiar que recibe el Adulto Mayor (Amigos)

\begin{tabular}{lcccccc}
\hline \multirow{2}{*}{ Tipo de apoyo que recibe } & \multicolumn{2}{c}{ HOMBRES } & \multicolumn{2}{c}{ MUJERES } & \multicolumn{2}{c}{ TOTAL } \\
\cline { 2 - 7 } & Frec. & $\%$ & Frec. & $\%$ & Frec. & $\%$ \\
\hline Dinero & 15 & 55,56 & 12 & 44,44 & 27 & 5,11 \\
Alojamiento & 1 & 16,67 & 5 & 83,33 & 6 & 1,14 \\
Comida & 12 & 30,00 & 28 & 70,00 & 40 & 7,58 \\
Ropa & 1 & 20,00 & 4 & 80,00 & 5 & 0,95 \\
Servicios & 1 & 33,33 & 2 & 66,67 & 3 & 0,57 \\
Cuidado & 25 & 21,55 & 91 & 78,45 & 116 & 21,97 \\
Transporte & 0 & 0 & 0 & 0 & 0 & 0,00 \\
Cuidados del hogar & 5 & 18,52 & 22 & 81,48 & 27 & 5,11 \\
Afecto & 50 & 27,32 & 133 & 72,68 & 183 & 34,66 \\
Compañía & 113 & 29,89 & 265 & 70,11 & 378 & 71,59 \\
Reconocimiento & 30 & 26,79 & 82 & 73,21 & 112 & 21,21 \\
Escucha & 66 & 26,94 & 179 & 73,06 & 245 & 46,40 \\
Intercambio de experiencias & 74 & 33,18 & 149 & 66,82 & 223 & 42,23 \\
Información & 56 & 30,11 & 130 & 69,89 & 186 & 35,23 \\
Consejos & 51 & 32,08 & 108 & 67,92 & 159 & 30,11 \\
\hline
\end{tabular}

El adulto mayor en contextos urbanos de pobreza, no solo es receptor de ayuda tanto de parte de su red familiar como de la no familiar, sino que también es proveedor de ayuda hacia las mismas, y en muy altas

proporciones, la tabla 12 permite apreciar que el $88,70 \%$ de los encuestados afirmó que otorga apoyo a sus amigos.

Tabla 12. Tipo de apoyo no familiar que recibe el Adulto Mayor (Amigos)

\begin{tabular}{lcccccc}
\hline \multirow{2}{*}{$\begin{array}{c}\text { Brinda } \\
\text { apoyo }\end{array}$} & \multicolumn{2}{c}{ HOMBRES } & \multicolumn{2}{c}{ MUJERES } & \multicolumn{2}{c}{ TOTAL } \\
\cline { 2 - 7 } & Frec. & $\%$ & Frec. & \% & Frec. & $\%$ \\
\hline SI & 155 & 88,07 & 363 & 88,97 & 518 & 88,70 \\
NO & 18 & 10,23 & 41 & 10,05 & 59 & 10,10 \\
NS / NR & 3 & 1,70 & 4 & 1 & 7 & 1,20 \\
TOTAL & $\mathbf{1 7 6}$ & $\mathbf{1 0 0}$ & $\mathbf{4 0 8}$ & $\mathbf{1 0 0}$ & $\mathbf{5 8 4}$ & $\mathbf{1 0 0}$ \\
\hline
\end{tabular}

En la tabla 13, se puede apreciar como a pesar de las condiciones de pobreza que caracterizan los adultos mayores que hacen parte de la muestra, los apoyos que estos brindan a sus amigos incluyen, aunque en una baja proporción, apoyo de tipo material como dinero en efectivo y comida; también expresaron que brindan cuidados a sus amigos en el $13,13 \%$ de los casos; los apoyos de tipo emocional son los que en mayor proporción se ofrecen, el 74,71\% expresa que apoya a sus amigos a partir de la compañía que ellos les brindan, el $40,15 \%$ brinda la posibilidad de escucharlos, el $38,61 \%$ intercambio de experiencias, el 38,03\% expresó brindar afecto a sus amigos, el 30,89\% información, el $27,22 \%$ consejo, y el $13,13 \%$ cuidados.

Tabla 13. Tipo de apoyo no familiar que brinda el Adulto Mayor (Amigos)

\begin{tabular}{lcccccc}
\hline \multirow{2}{*}{ Tipo de apoyo que brinda } & \multicolumn{2}{c}{ HOMBRES } & \multicolumn{2}{c}{ MUJERES } & \multicolumn{2}{c}{ TOTAL } \\
\cline { 2 - 7 } & Frec. & $\%$ & Frec. & $\%$ & Frec. & $\%$ \\
\hline Dinero & 6 & 37,50 & 10 & 62,50 & 16 & 3.09 \\
Alojamiento & 1 & 50,00 & 1 & 50,00 & 2 & 0.39 \\
Comida & 8 & 47,06 & 9 & 52,94 & 17 & 3.28 \\
Ropa & 1 & 100,00 & 0 & 0,00 & 1 & 0.19 \\
Servicios & 2 & 25,00 & 6 & 75,00 & 8 & 1.54 \\
Cuidado & 14 & 20,59 & 54 & 79,41 & 68 & 13.13 \\
Transporte & 0 & 0,00 & 2 & 100,00 & 2 & 0.39 \\
Hogar & 5 & 20,83 & 19 & 79,17 & 24 & 4.63 \\
Afecto & 45 & 22,84 & 152 & 77,16 & 197 & 38.03 \\
Compañía & 109 & 28,17 & 278 & 71,83 & 387 & 74.71 \\
Reconocimiento & 39 & 28,47 & 98 & 71,53 & 137 & 26.45 \\
Escucha & 54 & 25,96 & 154 & 74,04 & 208 & 40.15 \\
Intercambio experiencias & 68 & 34,00 & 132 & 66,00 & 200 & 38.61 \\
Información & 45 & 28,13 & 115 & 71,88 & 160 & 30.89 \\
Consejos & 41 & 29,08 & 100 & 70,92 & 141 & 27.22 \\
\hline
\end{tabular}

Rev. Invest. Univ. Quindío (22): 30-47. Armenia - Colombia 
El apoyo de tipo cognitivo también representa una de las principales fuentes de apoyo de los adultos mayores hacia sus amigos, se representa a través del intercambio de experiencias en el 38,61\% del total de la muestra, también ejerce la posibilidad de ser transmisor de información 30,89\% del total y de brindar consejos en el 27,22\%. (Tabla13).

Un resultado importante se evidencia en este misma tabla en relación con el género de los que ayudan, las mujeres presentan las mayores proporciones de apoyo hacia sus amigos o vecinos, de estos apoyos, los tipos más importantes en términos porcentuales son: Del
$74,71 \%$ del total que brinda compañía el $71,83 \%$ son mujeres, del 40,15 que brindan escucha lo son el $74.04 \%$, así secesivamente se puede apreciar que las mujeres en general son las que mayor cantidad de apoyo otorgan.

Desde el punto de vista de los adultos mayores entrevistados, la red no familiar brinda apoyo siempre en el $18,61 \%$ de los casos, en momentos de enfermedad en una proporción del $14,55 \%$, en situación de soledad en el 8,29\%; cuando se presentan calamidades en el $5,41 \%$ y cuando hay dificultades económicas en el $2,54 \%$ de los casos.

Tabla 14. Ocasiones en las que el A.M. recibe mayor apoyo de su red no familiar

\begin{tabular}{lcccccc}
\hline \multirow{2}{*}{$\begin{array}{c}\text { Ocasiones en que recibe } \\
\text { mayor apoyo }\end{array}$} & \multicolumn{2}{c}{ HOMBRES } & \multicolumn{2}{c}{ MUJERES } & \multicolumn{2}{c}{ TOTAL } \\
\cline { 2 - 7 } & Frec. & $\%$ & Frec. & $\%$ & Frec. & $\%$ \\
\hline Enfermedad & 26 & 30,23 & 60 & 69,77 & 86 & 14,55 \\
Muerte & 1 & 25,00 & 3 & 75,00 & 4 & 0,68 \\
Calamidad & 8 & 25,00 & 24 & 75,00 & 32 & 5,41 \\
Dificultades Económicas & 8 & 53,33 & 7 & 46,67 & 15 & 2,54 \\
Soledad & 12 & 24,49 & 37 & 75,51 & 49 & 8,29 \\
Siempre & 34 & 30,91 & 76 & 69,09 & 110 & 18,61 \\
Otro & 1 & 25,00 & 3 & 75,00 & 4 & 0,68 \\
NS / NR & 7 & 53,85 & 6 & 46,15 & 13 & 2,20 \\
\hline
\end{tabular}

Los adultos mayores presentan un apoyo de doble vía, mediante los que se demuestra reciprocidad entre el dar y el recibir, en relación con los momentos en que el A.M. presta mayor ayuda a los miembros de la red no familiar (amigos y vecinos) se ha encontrado que en orden de jerarquía coincide con los de la red familiar, aunque los porcentajes obtenidos en la red familiar fueron mucho mas altos, dichos eventos son en su orden: Siempre con $20,47 \%$, en la enfermedad con un $11,34 \%$; en momentos de soledad con un $7,78 \%$ y en situaciones de calamidad con un 5,25\%.

Tabla 15. Ocasiones en los que el A.M. brinda mayor apoyo a su red no familiar

\begin{tabular}{lcccccc}
\hline \multirow{2}{*}{$\begin{array}{c}\text { Ocasiones en que brinda } \\
\text { apoyo }\end{array}$} & \multicolumn{2}{c}{ HOMBRES } & \multicolumn{2}{c}{ MUJERES } & \multicolumn{2}{c}{ TOTAL } \\
\cline { 2 - 7 } & Frec. & $\%$ & Frec. & $\%$ & Frec. & $\%$ \\
\hline Enfermedad & 22 & 32,84 & 45 & 67,16 & 67 & 11,34 \\
Muerte & 2 & 100,00 & 0 & 0,00 & 2 & 0,34 \\
Calamidad & 10 & 32,26 & 21 & 67,74 & 31 & 5,25 \\
Dificultades Económicas & 1 & 25,00 & 3 & 75,00 & 4 & 0,68 \\
Soledad & 12 & 26,09 & 34 & 73,91 & 46 & 7,78 \\
Siempre & 37 & 30,58 & 84 & 69,42 & 121 & 20,47 \\
Otro & 3 & 25,00 & 9 & 75,00 & 12 & 2,03 \\
NS/ NR & 9 & 52,94 & 8 & 47,06 & 17 & 2,88 \\
\hline
\end{tabular}

En las proporciones obtenidas en la valoración de la satisfacción con la comunicación con los miembros de la red familiar, se ha encontrado que igualmente se manifiesta un alto nivel de satisfacción con la comunicación con los miembros de la red no familiar (amigos y vecinos), el $29,79 \%$ de los adultos mayores consideran tener un nivel muy alto de satisfacción; un $33,56 \%$ la consideran alta y el $33,56 \%$ no la considera ni alta ni baja. Se evidencia un contraste importante con los mas bajos niveles de satisfacción donde unicamente el 0,86\% expresó una baja satisfacción y 0,17 lo valoró como muy baja. 
Gómez S., C.A., Quintana U., L.I. - 41 -

Tabla16. Satisfacción con la comunicación con los miembros de la red no familiar

\begin{tabular}{lcccccc}
\hline \multirow{2}{*}{$\begin{array}{c}\text { Satisfacción con la } \\
\text { comuni cación }\end{array}$} & \multicolumn{2}{c}{ HOMBRES } & \multicolumn{2}{c}{ MUJERES } & \multicolumn{2}{c}{ TOTAL } \\
\cline { 2 - 7 } & Frec. & $\mathbf{\%}$ & Frec. & $\mathbf{\%}$ & Frec. & $\%$ \\
\hline 1.Muy alta & 46 & 26,14 & 128 & 31,37 & 174 & 29,79 \\
2.Alta & 62 & 35,23 & 134 & 32,84 & 196 & 33,56 \\
3.Ni alta ni baja & 59 & 33,52 & 137 & 33,58 & 196 & 33,56 \\
4.Baja & 1 & 0,57 & 4 & 0,98 & 5 & 0,86 \\
5.Muy baja & 1 & 0,57 & 0 & 0,00 & 1 & 0,17 \\
NS / NR & 7 & 3,98 & 5 & 1,23 & 12 & 2,05 \\
TOTAL & $\mathbf{1 7 6}$ & $\mathbf{1 0 0}$ & $\mathbf{4 0 8}$ & $\mathbf{1 0 0}$ & $\mathbf{5 8 4}$ & $\mathbf{1 0 0}$ \\
\hline
\end{tabular}

Las red social no familiar a partir de los datos, presentan una alta densidad / conectividad, pues en una mínima proporción se presenta la disolución de contactos entre sus miembros, por el contrario se aprecia su alta cohesión y estabilidad, representada aquí con el hecho de que el
95.94\%, no presenta disolución de contactos durante el último mes con ninguno de los miembros de la red no familiar. No se evidencian importantes diferencias por sexo.

Tabla 17. Disolución de contactos con los miembros de la red no familiar

\begin{tabular}{lcccccc}
\hline \multirow{2}{*}{$\begin{array}{c}\text { Disolución de } \\
\text { contactos }\end{array}$} & \multicolumn{2}{c}{ HOMBRES } & \multicolumn{2}{c}{ MUJERES } & \multicolumn{2}{c}{ TOTAL } \\
\cline { 2 - 7 } & Frec. & $\mathbf{\%}$ & Frec. & $\mathbf{\%}$ & Frec. & $\%$ \\
\hline Amigos & 5 & 2,15 & 3 & 0,84 & 8 & 1,35 \\
Vecinos & 5 & 2,15 & 5 & 1,40 & 10 & 1,69 \\
Grupos & 0 & 0,00 & 1 & 0,28 & 1 & 0,17 \\
Ninguno & 221 & 94,85 & 346 & 96,65 & 567 & 95,94 \\
NS / NR & 2 & 0,86 & 3 & 0,84 & 5 & 0,85 \\
TOTAL & $\mathbf{2 3 3}$ & $\mathbf{1 0 0}$ & $\mathbf{3 5 8}$ & $\mathbf{1 0 0}$ & $\mathbf{5 9 1}$ & $\mathbf{1 0 0}$ \\
\hline
\end{tabular}

Un aspecto que demuestra la conectividad es el hecho de que un $10,82 \%$ de los adultos mayores expresó haber iniciado un nuevo contacto ya sea con amigos $(5,92 \%)$, vecinos $(3,38 \%)$ o con grupos $(1,52 \%)$, pero resulta sorprendente observar que en 520 casos que representan un $87.98 \%$ del total, no han potenciado nuevos contactos, lo cual indica, teniendo en cuenta los contactos disueltos y los nuevos contactos, que la red no familiar presenta en términos generales una baja densidad y conectividad, lo cual limita la posibilidad de recibir nuevos apoyos a futuro.

Otro dato que vale la pena resaltar es que no se aprecia diferencias importantes entre la disolución y el establecimiento de contactos entre hombres y mujeres en esta red

Tabla18. Nuevos contactos con los miembros de la red no familiar

\begin{tabular}{lcccccc}
\hline & \multicolumn{2}{c}{ HOMBRES } & \multicolumn{2}{c}{ MUJERES } & \multicolumn{2}{c}{ TOTAL } \\
\cline { 2 - 7 } Nuevos contactos & Frec. & $\mathbf{\%}$ & Frec. & $\mathbf{\%}$ & Frec. & $\mathbf{\%}$ \\
\hline Amigos & 18 & 7,73 & 17 & 4,75 & 35 & 5,92 \\
Vecinos & 7 & 3,00 & 13 & 3,63 & 20 & 3,38 \\
Grupos & 1 & 0,43 & 8 & 2,23 & 9 & 1,52 \\
Ninguno & 204 & 87,5 & 316 & 88,27 & 520 & 87,99 \\
NS/ NR & 3 & 1,29 & 4 & 1,12 & 7 & 1,18 \\
TOTAL & $\mathbf{2 3 3}$ & $\mathbf{1 0 0}$ & $\mathbf{3 5 8}$ & $\mathbf{1 0 0}$ & $\mathbf{5 9 1}$ & $\mathbf{1 0 0}$ \\
\hline
\end{tabular}

\section{La red formal de apoyo}

En lo que respecta al apoyo social formal, se encontró que el $32,99 \%$ de los sujetos que conforman la muestra reciben ayuda de alguna institución, pero la gran mayoría, representada por el $64,30 \%$ no recibe ningún tipo de ayuda de parte de la red formal de apoyo, nuevamente son la mujeres las que en mayor proporción reciben ayuda, en este caso, ayuda formal, dado que de los que reciben ayuda el $37,75 \%$ son mujeres y solamente el $28,76 \%$ son hombres. (Tabla 19). 
Tabla 19. Apoyo formal recibido por adultos mayores

\begin{tabular}{lcccccc}
\hline \multirow{2}{*}{$\begin{array}{c}\text { Apoyo } \\
\text { institucional }\end{array}$} & \multicolumn{2}{c}{ HOMBRES } & \multicolumn{2}{c}{ MUJERES } & \multicolumn{2}{c}{ TOTAL } \\
\cline { 2 - 7 } \multicolumn{1}{c}{} & Frec. & \% & Frec. & \% & Frec. & $\%$ \\
\hline SI & 67 & 28,76 & 128 & 35,75 & 195 & 32,99 \\
NO & 157 & 67,38 & 223 & 62,29 & 380 & 64,30 \\
NS / NR & 9 & 3,86 & 7 & 1,96 & 16 & 2,71 \\
TOTAL & $\mathbf{2 3 3}$ & $\mathbf{1 0 0}$ & $\mathbf{3 5 8}$ & $\mathbf{1 0 0}$ & $\mathbf{5 9 1}$ & $\mathbf{1 0 0}$ \\
\hline
\end{tabular}

Como era de esperarse, las organizaciones de carácter público o gubernamental son las que en mayor proporción otorgan este tipo de ayuda, encontrandose un índice de $76,41 \%$, lo sigue de lejos las organizaciones de carácter comunitario con el $14,36 \%$ y las religiosas con el $6,15 \%$ de los casos y en un escaso $2,05 \%$ del apoyo formal formal es brindado por organizaciones no gubernamentales. (Tabla 20).

Tabla 20. Naturaleza de la institución que brinda apoyo

\begin{tabular}{lcccccc}
\hline \multirow{2}{*}{\begin{tabular}{c} 
Naturaleza de la \\
institución que brinda \\
\multicolumn{1}{c}{ apoyo }
\end{tabular}} & \multicolumn{2}{c}{ HOMBRES } & \multicolumn{2}{c}{ MUJERES } & \multicolumn{2}{c}{ TOTAL } \\
\cline { 2 - 7 } & Frec. & $\%$ & Frec. & $\%$ & Frec. & $\%$ \\
\hline Religión & 3 & 4,48 & 9 & 7,03 & 12 & 6,15 \\
Gubernamental & 54 & 80,60 & 95 & 74,22 & 149 & 76,41 \\
No gubernamental & 2 & 2,99 & 2 & 1,56 & 4 & 2,05 \\
Comunitaria & 6 & 8,96 & 22 & 17,19 & 28 & 14,36 \\
Otro & 2 & 2,99 & 0 & 0,00 & 2 & 1,03 \\
TOTAL & 67 & 100 & 128 & 100 & 195 & 100 \\
\hline
\end{tabular}

El tipo de ayuda o apoyo social formal que con mayor proporción reciben los adultos mayores pobres la constituye la ayuda en dinero en efectivo, en un $46,67 \%$ de los casos se encontró este tipo de apoyo,el 18,97\% de ellos recibe alimentos, el 14,36\% expresó recibir ayuda calificadas por ellos como de entretenimiento (actividades recreativas y celebraciones); el 12,82\% recibe compañía; el 11,28\% dijo recibir formación y el $10,26 \%$ capacitación, nuevos conocimientos 9,74\%. Llama poderosamente la atención de que esta ayuda formal además de la ayuda material, contempla acciones relacionadas con la adquisición de nuevos conocimientos y habilidades, así lo demuestran los tres últimos valores. (Tabla 21).

Tabla 21. Tipo de ayuda otorgada por la red formal.

\begin{tabular}{lcccccr}
\hline \multirow{2}{*}{ Tipo de ayuda } & \multicolumn{2}{c}{ 1. HOMBRE } & \multicolumn{2}{c}{ 2. MUJER } & \multicolumn{2}{c}{ TOTAL } \\
\cline { 2 - 7 } & Frec. & \% & Frec. & $\%$ & Frec. & \multicolumn{1}{c}{$\%$} \\
\hline Dinero & 33 & 36,26 & 58 & 63,74 & 91 & 46,67 \\
Alimentos & 14 & 37,84 & 23 & 62,16 & 37 & 18,97 \\
Ropa & 0 & 0,00 & 1 & 100,00 & 1 & 0,51 \\
Ayuda con cuidado personal & 0 & 0,00 & 6 & 100,00 & 6 & 3,08 \\
Entretenimiento & 9 & 32,14 & 19 & 67,86 & 28 & 14,36 \\
Compañía & 6 & 24,00 & 19 & 76,00 & 25 & 12,82 \\
Formación & 5 & 22,73 & 17 & 77,27 & 22 & 11,28 \\
Capacitación & 4 & 20,00 & 16 & 80,00 & 20 & 10,26 \\
Nuevos conocimientos & 6 & 31,58 & 13 & 68,42 & 19 & 9,74 \\
Otro & 13 & 30,23 & 30 & 69,77 & 43 & 22,05 \\
NS / NR & 1 & 100,00 & 0 & 0,00 & 1 & 0,51 \\
\hline
\end{tabular}

El hecho de que la mayoría de las instituciones que prestan ayuda al A.M. sean de naturaleza pública y que la mayor proporción de las ayudas esten representadas en dinero en efectivo y en alimentación, son un indicio claro que son los programas de protección social que está desarrollando el estado actualmente, los que 
constituyen la mayor fuente de apoyo formal para el adulto mayor, estos programas son el Programa de Protección Social al Adulto Mayor (PPSAM) ${ }^{6}$ con sus modalidades de ayuda en efectivo y ayuda de ración alimentaria y por otro lado el programa de alimentación complementaria Juan Luis Londoño De La Cuesta', coordinado por el ICBF.

La tabla 22, permite apreciar que una proporción importante de sujetos que reciben ayuda de las instituciones, tienen oportunidad de reciprocar al proveer ayuda a las instituciones que le ayudan a él $(56,92 \%)$, se confirma aquí como sucedió en la red informal, que el adulto mayor es una importante fuente de apoyo y de ayuda no solamente hacia sus familiares y amigos sino también hacia las instituciones y su comunidad y que también en el caso de la red formal son las mujeres las que en mayor proporción proveen ayuda. $(60,16 \%$ de las mujeres que brindan ayuda, apoyan a las instituciones comparado con el $50,75 \%$ de los hombres).

Tabla 22. Apoyo de los adultos mayores hacia la red formal

\begin{tabular}{lcccccc}
\hline \multirow{2}{*}{$\begin{array}{c}\text { Apoyo } \\
\text { institucional }\end{array}$} & \multicolumn{2}{c}{ HOMBRES } & \multicolumn{2}{c}{ MUJERES } & \multicolumn{2}{c}{ TOTAL } \\
\cline { 2 - 7 } & Frec. & $\%$ & Frec. & $\%$ & Frec. & $\%$ \\
\hline SI & 34 & 50,75 & 77 & 60,16 & 111 & 56,92 \\
NO & 33 & 49,25 & 51 & 39,84 & 84 & 43,08 \\
TOTAL & 67 & 100 & 128 & 100 & 195 & 100 \\
\hline
\end{tabular}

Las organizaciones de carácter comunitario, seguido por organizaciones de carácter religioso son las que en mayor proporción reciben apoyo de las personas adultas mayores con valores de $(33,33 \%)$ y $(22,05 \%)$, este aspecto se explica fácilmente al constatar que, al contrario de lo que se cree, las personas AM continúan participando de manera importante en algunos espacios sociales como grupos comunitarios gestionados por ellos mismos y por organizaciones de naturaleza religiosa donde realizan actividades de orden espiritual, de otra parte, una muy baja proporción, (1,03\%), expresaron brindar ayuda a las organizaciones de carácter público. (Tabla 23)

Las organizaciones de carácter comunitario están representadas aquí fundamentalmente por los clubes sociales que son autogestionados por los propios adultos mayores.

Tabla 23. Naturaleza de las organizaciones apoyadas por los A.M.

\begin{tabular}{lcccccc}
\hline \multirow{2}{*}{$\begin{array}{c}\text { Naturaleza de la } \\
\text { institución que apoya }\end{array}$} & \multicolumn{2}{c}{ HOMBRES } & \multicolumn{2}{c}{ MUJERES } & \multicolumn{2}{c}{ TOTAL } \\
\cline { 2 - 7 } & Frec. & $\%$ & Frec. & $\%$ & Frec. & $\%$ \\
\hline Religión & 12 & 17,91 & 31 & 24,22 & 43 & 22,05 \\
Gubernamental & 0 & 0,00 & 2 & 1,56 & 2 & 1,03 \\
No gubernamental & 0 & 0,00 & 1 & 0,78 & 1 & 0,51 \\
Comunitaria & 22 & 32,84 & 43 & 33,59 & 65 & 33,33 \\
NS/NR & 33 & 49,25 & 51 & 39,84 & 84 & 43,08 \\
TOTAL & $\mathbf{6 7}$ & $\mathbf{1 0 0}$ & $\mathbf{1 2 8}$ & $\mathbf{1 0 0}$ & $\mathbf{1 9 5}$ & $\mathbf{1 0 0}$ \\
\hline
\end{tabular}

\footnotetext{
${ }^{6}$ El -PPSAM- tiene como objetivo fundamental proteger al adulto mayor, que se encuentra en estado de indigencia o de extrema pobreza, contra el riesgo económico de la imposibilidad de generar ingresos y contra el riesgo derivado de la exclusión social. Con base en la Ley $797 / 03$, la Ley 100 de 1993 , el Decreto 2681/03 (derogado por el decreto 569/04 y modificado por el decreto 4112/04) y el Conpes Social 70 de Mayo $28 / 03$, (y los Conpes complementarios 78 de Mayo 3/04 y el 082 de Septiembre 6/04), se diseñó el Programa de Protección Social al Adulto Mayor, -PPSAM- donde el Ministerio de la Protección Social es quien lidera la planeación, programación y ejecución del mismo.

${ }^{7}$ El objetivo del programa es brindar alimentación complementaria mediante un almuerzo durante 250 días al año, a 400.000 adultos mayores en condiciones de extrema vulnerabilidad económica y social. Existen dos modalidades de atención: 1 . Consiste en el suministro de un almuerzo preparado y servido localmente, para adultos mayores ubicados en el área urbana o en el área rural concentrada siempre y cuando haya disponibilidad de infraestructura para la preparación del mismo y; 2 . Suministro mensual de un paquete alimentario con productos no perecederos, para adultos mayores ubicados en área rural dispersa, o en zonas rurales concentradas donde no exista infraestructura para la preparación del almuerzo caliente.
} 
A pesar de las precarias condiciones de vida que presenta la población objeto de este trabajo se encontró que de los adultos mayores que prestan ayuda, brindan apoyo de carácter material, así: el $30,97 \%$ apoya económicamente a las instituciones que a su vez le ayudan a él, el 28,32\% apoya con alimentos, el $12,39 \%$ ayuda con ropa. Por otro lado un muy importante porcentaje de $43,36 \%$ de todos los que ayudan a las organizaciones lo hacen a través de voluntariados o trabajo voluntario, otros valores dignos de tener en cuenta lo constituyen los apoyos que se expresan a través de compañía en un $16,81 \%$ de los casos y de entretenimiento en el $15,93 \%$ de los casos. (Tabla 24)

Tabla 24. Tipo de ayuda otorgada a la red formal de parte del A.M.

\begin{tabular}{lcccccc}
\hline \multirow{2}{*}{ Tipo de apoyo que ofrec } & \multicolumn{2}{c}{ HOMBRE } & \multicolumn{2}{c}{ MUJER } & \multicolumn{2}{c}{ TOTAL } \\
\cline { 2 - 7 } & Frec. & $\%$ & Frec. & $\%$ & Frec. & $\%$ \\
\hline Dinero & 10 & 28,57 & 25 & 71,43 & 35 & 30,97 \\
Comida & 11 & 34,38 & 21 & 65,63 & 32 & 28,32 \\
Ropa & 5 & 35,71 & 9 & 64,29 & 14 & 12,39 \\
Ayuda con oficios del hogar & 0 & 0,00 & 3 & 100,00 & 3 & 2,65 \\
Ayuda con cuidado personal & 0 & 0,00 & 8 & 100,00 & 8 & 7,08 \\
Entretenimiento & 2 & 11,11 & 16 & 88,89 & 18 & 15,93 \\
Compañía & 4 & 21,05 & 15 & 78,95 & 19 & 16,81 \\
Trabajo voluntario & 18 & 36,73 & 31 & 63,27 & 49 & 43,36 \\
Otro & 0 & 0,00 & 6 & 100,00 & 6 & 5,31 \\
\hline
\end{tabular}

La periodicidad con que el adulto mayor recibe ayudas de parte de la red formal es variada, el 45,13\% de los encuestados recibe ayuda mensualmente (presumiblemente los beneficiarios de los programas PPSAM y PPNAM), el14,36\% lo hace semanalmente y el $10,26 \%$ recibe ayuda diaria, (Tabla 25 ).

Tabla 25. Frecuencia con que recibe ayuda de la red formal

\begin{tabular}{lcccccc}
\hline & \multicolumn{2}{c}{ HOMBRE } & \multicolumn{2}{c}{ MUJER } & \multicolumn{2}{c}{ TOTAL } \\
\cline { 2 - 7 } \multicolumn{1}{c}{ Frecuencia } & Frec. & \% & Frec. & $\%$ & Frec. & \% \\
\hline Diario & 7 & 10,45 & 13 & 10,16 & 20 & 10,26 \\
Semanal & 7 & 10,45 & 21 & 16,41 & 28 & 14,36 \\
Mensual & 30 & 44,78 & 58 & 45,31 & 88 & 45,13 \\
Anual & 4 & 5,97 & 2 & 1,56 & 6 & 3,08 \\
Eventualmente & 7 & 10,45 & 19 & 14,84 & 26 & 13,33 \\
NS/ NR & 12 & 17,91 & 15 & 11,72 & 27 & 13,85 \\
TOTAL & $\mathbf{6 7}$ & $\mathbf{1 0 0}$ & $\mathbf{1 2 8}$ & $\mathbf{1 0 0}$ & $\mathbf{1 9 5}$ & $\mathbf{1 0 0}$ \\
\hline
\end{tabular}

El A.M. de las zonas urbanas en condición de pobreza ayuda a las organizaciones que hacen parte de la red formal eventualmente en el $24,10 \%$ de los casos, mensualmente en el $7,18 \%$, semanalmente en el $17,44 \%$ de los casos y diariamente en el $6,15 \%$ de los casos. Podría pensarse que son apoyos poco significativos pero en virtud de sus desventajosas condiciones de vida podemos concluir que son apoyos muy importantes en relación con sus posesiones y consecuente posibilidad de dar.

Tabla 26. Frecuencia con que el A.M. otorga ayuda a la red formal

\begin{tabular}{lcccccc}
\hline & \multicolumn{2}{c}{ 1. HOMBRE } & \multicolumn{2}{c}{ 2. MUJER } & \multicolumn{2}{c}{ TOTAL } \\
\cline { 2 - 7 } Frecuencia & TOTAL & \% & TOTAL & \% & TOTAL & \% \\
\hline Diario & 3 & 4,48 & 9 & 7,03 & 12 & 6,15 \\
Semanal & 8 & 11,94 & 26 & 20,31 & 34 & 17,44 \\
Mensual & 4 & 5,97 & 10 & 7,81 & 14 & 7,18 \\
Anual & 0 & 0,00 & 1 & 0,78 & 1 & 0,51 \\
Eventualmente & 19 & 28,36 & 28 & 21,88 & 47 & 24,10 \\
NS / NR & 33 & 49,25 & 54 & 42,19 & 87 & 44,62 \\
TOTAL & $\mathbf{6 7}$ & $\mathbf{1 0 0}$ & $\mathbf{1 2 8}$ & $\mathbf{1 0 0}$ & $\mathbf{1 9 5}$ & $\mathbf{1 0 0}$ \\
\hline
\end{tabular}

Rev. Invest. Univ. Quindío (22): 30-47. Armenia - Colombia 


\section{CONCLUSIONES}

Los datos presentados son contundentes en relación con la función del Adulto Mayor Pobre en el ejercicio del apoyo social familiar y no familiar, se desvirtúa la creencia de que éste es un agente pasivo que se limita simplemente a recibir ayuda de la red familiar y la no familiar, es claro que los adultos mayores son proveedores de ayuda a los demás miembros de sus redes, incluso se ha encontrado que en algunos casos, brinda ayuda en mayores proporciones comparado con los apoyos recibidos. Paralelamente esto permite constatar que existe reciprocidad (dar y recibir) tanto en la red familiar como en la no familiar y la formal, en la red social del A.M.P. del departamento del Quindío.

En este orden de ideas, este ejercicio investigativo permite concluir que las redes sociales del Adulto Mayor pobre (A.M.P), juegan un rol determinante en relación con el apoyo que reciben las personas más viejas de parte tanto de la red familiar como de la no familiar, los datos demuestran que la gran mayoría de ellos recibe algún tipo de apoyo y son excepcionales los casos en que, teniendo vínculos con familiares o con amigos y vecinos, no recibe ayudas, lo cual demuestra una vez más cómo la solidaridad y el apoyo mutuo reemplazan o mitigan la ausencia de los recursos necesarios que les permitan acceder a los bienes $y$ servicios que requieren para subsistir, una cosa es que cuantitativamente el tamaño de la red sea limitado y otra que cualitativamente la red juega un papel decisivo en el bienestar del Adulto Mayor Pobre.

Las difíciles condiciones de vida que enfrentan los adultos mayores y sus redes (familiares y no familiares) limitan de manera importante los intercambios y ayudas de carácter material que se dan entre ellos, a pesar de ello, se evidencia un importante flujo de ayudas representadas en dinero en efectivo, alimentación, alojamiento y en ropa. El tipo de apoyo que en mayor proporción recibe esta población la representa fundamentalmente los apoyos emocionales tales como afecto, compañía, escucha.

El adulto mayor por su parte, brinda básicamente apoyos de tipo emocional (afecto, compañía, escucha) y también cumple un papel importante en relación con los apoyos de carácter instrumental, al proporcionar cuidados (a los niños, enfermos y cuidado del hogar) a los miembros de las redes que lo requieren.
Los intercambios se presentan regularmente, no es necesario que exista una condición especial para que éstos se efectúen, aunque la situación de enfermedad del adulto mayor o de algún otro miembro de la red, es un aspecto importante a tener en cuenta ya que sugiere que es una variable que propicia situaciones de intercambio de bienes y/o servicios hacia el individuo enfermo.

Las redes sociales en los A.M.P. cumplen una función básica en relación con apoyos de carácter cognitivo, este recibe y provee información a través de intercambio de experiencias, de información y sobre todo de consejos que le permiten afrontar situaciones adversas, derivadas de sus precarias condiciones de vida.

Una característica importante en las redes sociales de la población A.M.P. es que a pesar de su limitado tamaño, los vínculos y apoyos que se dan en su interior son estables y con una fuerte cohesión, existe poca tendencia a la disolución de contactos y por el contrario estas redes representan una oportunidad para potenciar nuevas relaciones con vecinos, amigos y con instituciones o grupos.

La red formal de apoyo a la que tiene acceso la población A.M.P. es extremadamente "pobre", una muy limitada proporción de A.M. reciben apoyo por parte de Instituciones o de redes de atención profesional y de voluntariados, la participación en organizaciones comunitarias autogestionadas por los propios adultos mayores es relativamente baja, son las organizaciones de carácter oficial, público o gubernamental las que fundamentalmente, constituyen esta red.

La naturaleza del apoyo recibido de parte de la red formal, la constituyen básicamente los apoyos en dinero y en alimentación, lo cual indica que esta red se limita a proveer servicios eminentemente de carácter asistencial (estos A.M. son los beneficiarios del Programa de Protección Social al Adulto Mayor PPSAM y al Programa Nacional de Alimentación PPNAM). Este tipo de programas son necesarios, teniendo en cuenta las condiciones de vida de la población estudiada, pero es claro que son abiertamente insuficientes dada su escasa cobertura en relación con la demanda. De otro lado es importante promover una amplitud de esta red en terminos de generar y orientar los esfuerzos para ampliar las posibilidades de acción y de inserción social de los A.M.P.

Rev. Invest. Univ. Quindío (22): 30-47. Armenia - Colombia 
En general no se perciben diferencias importantes en la estructura y funcionamiento de las redes sociales de adultos mayores en función del sexo ni en la red familiar ni en la informal, solamente se aprecia que la mujer recibe más apoyo que el hombre de parte de la red formal, los programas de protección social privilegian en mayor medida a las mujeres que a los hombres.
Los resultados de este trabajo permiten concluir que las precarias condiciones socioeconómicas que caracterizan a la población estudiada limitan enormemente el adecuado funcionamiento de las redes sociales en la población adulta mayor, esto se refleja en el limitado tamaño de las redes

\section{BIBLIOGRAFÍA}

Barnes, J. A.1954. "Class committee in a Norwegian island parish", En: Human relations, 7, pp. 98-99. Bott. E. 1975. Family and Social Network. Londres. Tavistock.

Bott, E. 1980. Familias urbanas: Papeles conyugales y redes sociales. En: Sociología de la Familia. Selección de Michael Anderson. Fondo de Cultura Económica.

Bott. E. 1990. Familia y red social. Roles normas y relaciones externas en las familias urbanas corrientes. Taurus Humanidades. Madrid.

Chappel, N. 1992. Social Support and Agging, Butterworths Perspectives on individual and Population Agging Series. Canada. Pp.105.

Dabas, Elina. 1993. Red de redes. Las prácticas de la intervención en redes sociales. Argentina. Paidós.

De la Peña, G. 2001. Presentación Larissa Adler Lomnitz, antropóloga latinoamericana. En Lomnitz Larissa Adler, Redes Sociales cultura y poder. Ensayos de Antropología Latinoamericana. FLACSO-México. Ángel Porrúa 9-18.

Giraldo, Néstor; De La Rosa John y Fernández Álvaro (2008). "Dependencia económica y social de adultos mayores de 60 años en la ciudad de Armenia, Quindío". En: Revista de investigaciones Universidad del Quindío. No. 18. Armenia, Quindío.

Gómez, Cesar A. (2008). "Vejez y pobreza en el Departamento del Quindío". En: Revista de investigaciones Universidad del Quindío. No. 18. Armenia, Quindío.

González de la Rocha, Mercedes (1999). "La reciprocidad amenazada: Un costo más de la pobreza urbana", en Enríquez, Rocío, Hogar, pobreza y bienestar en México, Centro de Investigación y Formación Social, ITESO, México, 13-36.

Gottlieb B. (1983) Social networks and social support. Beverly Hills, CA: Sage.

Guzmán, José Miguel, Huenchuan Sandra y Montes de Oca Verónica. 2003. "Redes de apoyo social de personas mayores: marco teórico conceptual" CELADE-División de Población de la CEPAL Universidad Nacional Autónoma de México, México. Ponencia presentada en el Simposio Viejos y Viejas. Participación, Ciudadanía e Inclusión Social 51 Congreso Internacional de Americanistas Santiago de Chile, 14 al 18 de julio de 2003.

JOHNSON, Paul, 2002. Long-Term Historical Changes in the Status of Elders: Britain as an Exemplar of Advanced Industrial Economies. London School of Economics presentado en UNRISD Meeting on Ageing, Development and Social Protection. Madrid.

Khan, R.L. y Antonucci Toni (1980). Convoys over de life course: attachment, roles and social support. En P.B. Baltes y O. Brim. (eds). Life-span development a behavior, Vol. 3. pp 254-283. Boston. Lexington.

LASLETT, Peter, 1995. "Necessary Knowledge: Age and Aging in the Societies of the Past" in Aging in the Past Demography, Society and Old Age. Ed. by David Kertzer and Peter Laslett. The University of California Press. Scholarship Editions. U.S.A. 
Lindon; Siles y Schmid. 2003. "Capital social y pobreza: el marco analítico". En: Capital social y reducción de la pobreza en América Latina: en busca de un nuevo paradigma. Naciones Unidas, CEPAL, Universidad del Estado de Michigan. Santiago de Chile.

Lomnitz, Larissa, 1994. Redes sociales, cultura y poder: Ensayos de Antropología Latinoamericana, México: Flacsogrupo editorial Miguel Ángel Porrúa.

Lomnitz, Larissa. 1975. ¿Cómo sobreviven los marginados? Ed. Siglo XXI, México.

Lopata H. (1979) Women ad widows: support systems. Nueva York.: Elsevier.

Mingione, E. 1994. Sector informal y estrategias de sobrevivencia: hipótesis para el desarrollo de un campo de indagación. En Milán René (Comp.) Solidaridad y producción informal de recursos, Instituto de Investigaciones Sociales UNAM, (Mexico).

Minois, Georges (1987). “Historia de la vejez: De la antigüedad al renacimiento”. Madrid. Nerea

Scott, Anne y Wenger Clare. 1996. "Género y redes de apoyo social en la vejez". En: Arber, Sara y Jay Ginn, Relación entre género y envejecimiento. Enfoque sociológico, Ed. Narcea, Madrid. 